\title{
Sistema de Recomendação de Links para o fomento de discussões em fóruns de um Ambiente Virtual de Aprendizagem
}

\author{
Luis Carlos Costa Fonseca, UEMA, lccfonseca@gmail.com \\ Marlon Pereira Farias, UEMA, marlon.uema@gmail.com \\ Reinaldo de Jesus da Silva, UEMA, reinaldo.silvarrrb@gmail.com
}

\begin{abstract}
Resumo: Os fóruns de discussão apresentam-se como umas das ferramentas de interação mais utilizadas nos ambientes virtuais de aprendizagem. São objetos de estudo de várias pesquisas em informática na educação, tanto no que se refere a sua melhor utilização, como na avaliação de seus registros. Neste trabalho é proposta uma ferramenta que identifica palavras-chaves relevantes, através de técnicas de mineração textual, e posteriormente às submete a um motor de busca da Internet para recuperar documentos correlacionados aos assuntos do fórum. Pretende-se com isto, fomentar os debates através da apresentação de referencial textual extraído da rede mundial de computadores, e dessa forma propor uma recomendação de informações baseada em conteúdo.
\end{abstract}

Palavras-chaves: Recomendação de informação, Mineração de texto, tf*pdf.

\section{Links Recommendation System for the promotion of discussions on forums of a Virtual Learning Environment}

\begin{abstract}
Discussion forums present themselves as one of the most popular interaction tools in learning management systems. Many researchers in computer science education study them, both concerning their best use, such as assessment of their records. This paper proposes a tool that identifies relevant keywords via text mining techniques, and then submit it to a search engine on the Internet to retrieve documents related to the matters of the forum. It is intended with this, foment debate by presenting extracted textual reference from the World Wide Web, and thus propose a content-based recommendation of information.
\end{abstract}

Keywords: Recommendation systems, Text mining, tf*pdf.

\section{INTRODUÇÃO}

Qual o melhor caminho para chegar ao centro na hora do rush? Aonde se pode encontrar um bom restaurante nestas proximidades? Qual a melhor época para viajar para o caribe? Qual a melhor empresa para investir em ações? Qual computador comprar que atenda às necessidades específicas de um determinado usuário?

Tomar decisões é algo corriqueiro na vida das pessoas, para isto, faz-se uso de diversas técnicas que apoiem este processo, como por exemplo: pedir ajuda a um amigo, fazer uma consulta na Internet, contratar um especialista da área, entre outros. Nestas situações, o que se deseja é um meio pelo qual possa auxiliar a realizar boas decisões, ou seja, uma recomendação.

O ser humano está acostumado a pedir recomendações. Esta atividade é tão comum e frequente, que se viu a possibilidade de se realizar recomendações automáticas através de sistemas computacionais. Empresas como Amazon, NetFlix, Ebay, Youtube, Google, Yahoo, entre outras, se destacaram por fazer uso destes sistemas e investir no desenvolvimento de novos métodos relacionados a esta 
tecnologia. Ricci (et al.,2011) apresenta os Sistemas de Recomendação como ferramentas de software e técnicas que fornecem sugestões de itens que sejam úteis para o usuário.

A utilização de Sistemas de Recomendação tem crescido bastante desde o seu surgimento na década de 90 . Desde então, muito se fez na criação de novos métodos e abordagens que permitam a utilização desta tecnologia nas mais diversas áreas, onde os sistemas de comércio eletrônico se destacam entre os demais como os principais utilizadores desta tecnologia, de tal forma que já é parte fundamental da maior parte destes sistemas.

A Educação a Distância (EaD) passou por diversas mudanças nos últimos 20 anos. O advento da Internet da década de 90 permitiu uma mudança muito grande na EaD. O uso das Tecnologias de Informação e Comunicação (TIC), principalmente as relacionadas à Internet, possibilitou que os participantes (professores e alunos) do processo de ensino e aprendizagem pudessem interagir. Piva (et. al., 2011) afirma que essa evolução provocou uma mudança de paradigma no sentido que a individualização cedeu lugar à colaboração. E acrescenta que a aprendizagem independente passou a ser sustentada por experiências colaborativas entre alunos e professores e entre alunos.

O Ambiente Virtual de Aprendizagem (AVA) é um espaço online destinado a organizar e coordenar as atividades de ensino, ou seja, são as salas de aula online. Este ambiente é composto por diversas ferramentas como os chats, as videoconferências, os fóruns, entre outros. Tais instrumentos auxiliam o processo de interação e buscam tornar o processo de aprendizado mais atrativo para o aluno. Uma das principais ferramentas na construção do conhecimento nos AVA são os fóruns. Os fóruns são ferramentas de discussão e troca de ideias, auxiliando na construção coletiva do conhecimento e na integração entre alunos e professores. Trata-se de um espaço interativo assíncrono para a troca de mensagens permitindo a todos os participantes trocarem opiniões e informações. Os fóruns de discussão são uma das principais ferramentas na construção de conhecimento colaborativo nos AVA, são também objetos de estudo de várias pesquisas em informática na educação, tanto no que se refere a sua melhor utilização, como na avaliação de seus registros.

PIVA (2011, apud FERNANDEZ, 2013) tece uma crítica aos AVA's, pois afirma que ele não é capaz de, adequadamente, manter processos de aprendizado que permitam, além de um retorno automático aos alunos, um direcionamento do processo de aprendizado.

Diante do exposto acima, este artigo propõe a incorporação de um sistema de recomendação de links aos Ambientes Virtuais de Aprendizagem (AVA) com o intuito de fomentar discussões em fóruns de aprendizagem, desta forma, criando uma dinâmica que proponha novas leituras relacionadas ao tema da discussão.

Este trabalho está construído em sete seções dispostos da seguinte forma: Os conceitos relacionados às tecnologias são apresentados nas seções 2 e 3 . A seção 4 apresenta a descrição do problema que este trabalho se propõe a solucionar. Na seção 5 é feita a demonstração do sistema implementado. Na seção 6 é delineada a pesquisa realizada e os seus resultados. Por fim, na seção 7, são apresentadas as conclusões.

\section{SISTEMAS DE RECOMENDAÇÃO}

O ser humano hoje vive na era da informação, os computadores e as redes de telecomunicações foram grandes responsáveis por diversas mudanças sócio econômicas no século XX. Neste cenário nos deparamos com uma carga de informações cada vez 
maior. Os slogans afirmam que o mundo está a apenas um clique de distância. Mas será que isto é verdade?

Ainda há falta de informação, só que agora o problema se apresenta de uma forma diferente, falta informação relevante. A grande quantidade de conteúdo acaba gerando uma sobrecarga de informação que pode ser vista facilmente ao navegar pela Internet. Isto acaba desestimulando o usuário, pois há dificuldade em encontrar o que se realmente deseja, como consequência, o usuário se sente perdido em meio a um mundo de informações sem saber como encontrar o que lhe interessa. Mas surge a questão: como encontrar o conteúdo de interesse do usuário em meio a tanta informação?

Os sistemas de recomendação surgiram como uma resposta à dificuldade das pessoas de escolher em meio a grande variedade de produtos e serviços e as várias alternativas apresentadas.

Sistemas de Recomendação são ferramentas de software e técnicas que fornecem sugestões de itens que sejam úteis para um usuário. Segundo Ricci (et al., 2011) os Sistemas de Recomendação tentam prever quais são os produtos ou serviços mais adequados, com base nas preferências e restrições do usuário. Para completar esta tarefa, os sistemas de recomendação coletam dos usuários suas preferências, e que podem ser explicitas, através das avaliações de produtos, ou implícitas, inferidos através da interpretação das ações do usuário.

Os sistemas de recomendação surgiram para auxiliar no processo de indicar e receber indicações, desta forma, procuram facilitar a busca por conteúdo interessante ao usuário prevendo itens que possam ser relevantes ao mesmo.

Segundo Ricci (et al., 2011) a fim de implementar a sua função principal, identificando os itens úteis para o usuário, o sistema de recomendação deve predizer que item vale a pena recomendar. Para isto, o sistema deve ser capaz de prever a utilidade de alguns deles, ou, pelo menos, comparar a utilidade de alguns dos itens e, então decide quais os itens recomendar com base nesta comparação.

Sistemas de Recomendação envolve a construção de um modelo ou perfil de interesses do usuário. A construção deste perfil varia de acordo com a técnica de recomendação utilizada tendo como as principais técnicas de recomendação a Filtragem Colaborativa, Recomendação Baseada em Conteúdo, O modelo Híbrido, entre outros.

\section{MINERAÇÃO DE TEXTO}

Os avanços tecnológicos na área de compartilhamento e armazenamento de dados fizeram com que o volume de informações no formato digital crescesse em proporções antes inimagináveis. Segundo Kuechler (2007) 80\% desses dados não estão em formato estruturado, sendo que uma grande parte deles são textos. Essas informações incluem: e-mails, arquivos eletrônicos gerados por softwares editores de texto, páginas web, campos textuais em banco de dados, etc. Entretanto, esse formato de documentos foi criado para serem visualizados por seres humanos e não são adequados para a manipulação das informações neles contidas por sistemas computacionais.

Em geral, estes conteúdos são muito relevantes para as organizações, pois segundo Han e Kamber (2006), constituem um importante repositório organizacional, que envolve o registro de histórico de atividades, memorandos, documentos internos, emails, projetos, estratégias e o próprio conhecimento adquirido. Wives (2002) afirma que esse tipo de informação é muito importante para que os empresários consigam identificar novos dados e conhecimentos que estejam, de alguma forma, implícitos ou escondidos nos seus Sistemas de Informação e que não possam ser recuperados pelos meios tradicionais de recuperação oferecidos por eles. 
Para Rezende, Marcacini e Moura (2011), a organização inteligente dessas coleções textuais é de grande interesse para a maioria das instituições, pois agiliza os processos de busca e recuperação da informação. Entretanto, o volume de dados textuais armazenados é tal que extrapola a capacidade humana de, manualmente, analisá-lo e compreendê-lo por completo.

A mineração de textual surgiu da necessidade de se descobrir, de forma automática, informações em documentos, onde, segundo Aranha e Passos (2006), o uso dessa tecnologia permite recuperar informações, extrair dados, resumir, descobrir padrões, associações e regras e realizar análises qualitativas ou quantitativas em documentos de texto.

A mineração de textos, também chamado de mineração de dados textuais, permite transformar grande parte desses conteúdos não estruturados em conhecimento útil para as organizações.

Konchady (2006) apresenta uma definição geral de mineração de texto que inclui todos os tipos de processamento de texto que tratam de encontrar, organizar e analisar informações.

Lopes (2004) apresenta o seguinte conceito: Text mining, também conhecido como Text data mining ou Knowledge Discovery from textual databases, refere-se ao processo de extrair padrões interessantes e não triviais ou conhecimento a partir de documentos em textos não-estruturados. Text mining pode também ser definido como um conjunto de técnicas e processos que se prestam a descobrir conhecimento inovador nos textos. Esta nova tecnologia está sendo empregada, atualmente, em projetos de diversas áreas.

Desta forma, podemos entender a mineração de texto como sendo a aplicação de um conjunto de diferentes técnicas em dados não estruturados com o objetivo de obter informações, que muitas das vezes, podem não estar presente de maneira explicita nestes documentos. Inspirado pelo Data Mining ou mineração de dados, que procura descobrir padrões emergentes em banco de dados estruturados, a mineração de textos tem como objetivo a extração de conhecimento úteis em dados não estruturados ou semiestruturados.

Para Konchady (2006) tanto mineração de dados quanto mineração de texto buscam por informações escondidas e empregam algoritmos semelhantes de Inteligência Artificial, aprendizagem de máquina, e estatística. Mas enquanto a mineração de dados lida com dados estruturados, mineração de texto lida com dados não estruturados. Isto é, uma extensão da área de Data Mining focada na análise de textos.

Wives (2002) apresenta o processo de descoberta de conhecimento em textos como uma evolução natural da recuperação de informações, já que os sistemas de recuperação de informação passaram a adotar algumas técnicas de análise de informações e de aprendizado de máquina, muitas das quais provenientes da área de descoberta de conhecimento em bases de dados. Assim, ao invés do usuário ter que analisar quais dos documentos retornados são realmente relevantes, o próprio sistema faria essa análise e retornaria as informações de forma condensada e resumida.

\section{DESCRIÇÃO DO PROBLEMA}

O surgimento e a expansão da Internet na década de 90 foi responsável por profundas mudanças na educação a distância. O uso das Tecnologias de Informação e Comunicação (TICs) trouxeram um novo formato em que professores e alunos pudessem interagir. 
Tais mudanças permitiram uma nova dinâmica onde segundo (PIVA et al.,2011) provocou uma mudança de paradigma no sentido que a individualização cedeu lugar à colaboração e a aprendizagem independente passou a ser sustentada por experiências colaborativas entre alunos e professores e alunos entre si.

Uma das principais ferramentas dos AVAs voltadas a construção do conhecimento de forma colaborativa são os Fóruns de Discussões. Os fóruns são espaços de discussões e troca de ideias em torno de temas propostos por seus participantes. Este instrumento permite que cada participante submeta sua colaboração referente ao tema proposto, buscando assim o entendimento mútuo. Segundo Silva (2006, apud Okada) o fórum é uma ferramenta assíncrona que representa um espaço para debates no qual pode ocorrer o entrelaçamento de muitas vozes para a construção e desconstrução de pensamentos, para questionar e responder dúvidas, trilhando novos caminhos para a aprendizagem.

Sobre a importância destes debates Kenski (2002) traça o seguinte comentário:

\begin{abstract}
Interagir com o conhecimento e com as pessoas para aprender é fundamental. Para a transformação de um determinado grupo de informações em conhecimentos é preciso que estes sejam trabalhados, discutidos, comunicados. As trocas entre colegas, os múltiplos posicionamentos diante das informações disponíveis, os debates e as análises críticas auxiliam a sua compreensão e elaboração cognitiva. As múltiplas interações e trocas comunicativas entre parceiros do ato de aprender possibilitam que estes conhecimentos sejam permanentemente reconstruídos e reelaborados.
\end{abstract}

Com relação a participação e envolvimento nos fóruns de discussões Oliveira (2005) salienta que a participação no espaço criado pelo fórum pede preparo, geralmente provido por leituras adequadas, pesquisas, resgates ao background próprio a cada participante, entre outras formas de busca. Trata-se de organizar o pensamento, enriquecendo-o com pertinentes referências, permitindo o uso do espaço de discussões e reflexões proporcionado pelo fórum para gerar colaborações e agregar ideias.

Partindo deste problema, esta pesquisa propõem a utilização de um sistema com o objetivo de fomentar discussões em fóruns por meio da recomendação de links. Os conceitos e arquitetura deste sistema são apresentados no tópico a seguir.

\title{
5 DESCRIÇÃO DO SISTEMA
}

Como se pode perceber, todas as tecnologias apresentadas acima estão voltadas a manipulação, organização e busca de informação, mas não de qualquer tipo de informação, mas sim de informação relevante e útil para o usuário.

Para que isto seja possível, teria de se fazer uso de uma ferramenta de mineração de texto para a identificação das palavras chaves que representem os tópicos do fórum. Uma vez identificadas, estas palavras são submetidas a um motor de busca que retornará os links que irão oferecer novos conteúdos ao usuário para se aprofundar no contexto da discussão. Estes links serão avaliados pelo usuário e alimentarão um perfil que no futuro fará novas sugestões baseados em gostos anteriores do usuário.

A imagem a seguir apresenta o fluxograma que representa a arquitetura do sistema proposto. 


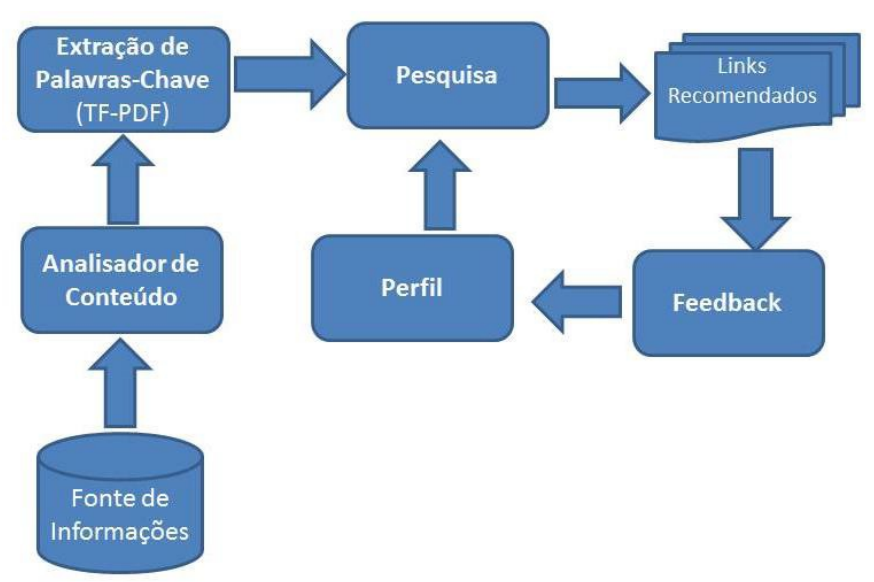

Figura 1: Arquitetura do Sistema

O sistema proposto neste trabalho busca recomendar links com o objetivo de fomentar discussões em AVA, desta forma, a recomendação de links busca oferecer novas possibilidade de leituras que venha desenvolver novas ideias e aprimorar o conhecimento.

1. O processo de recomendação de links tem início através da análise e processamento das informações. Neste caso, a fonte de informações são os tópicos (discussões) do fórum. Cada tópico do fórum é representado por um arquivo (.txt).

2. O analisador de conteúdo consiste no pré-processamento do texto dos fóruns. Como os conteúdos dos fóruns se encontram em linguagem natural, antes de se aplicar qualquer método estatístico de cálculo de relevância de termos, é feita uma triagem para separar os termos não representativos (stopwords), como advérbios, adjetivos, artigos e preposições, ou seja, são termos que em geral não acrescentam representatividade ao documento, desta forma os termos do documento que estão presente nesta lista de stopword são desconsiderados (eliminados) na representação do documento.

3. A ponderação de termos (Term Weighting) é uma importante ferramenta para determinar a relevância de uma palavra. A principal abordagem utilizada para o cálculo de peso de um termo é o proposto por Salton (1988) TF*IDF. Onde TF é igual a:

$$
\mathrm{TF}\left(t_{k}, d_{j}\right)=\frac{f_{k, j}}{\max _{z} f_{z, j}}
$$

Onde o máximo é calculado sobre as frequências fz,j de todos os termos tz que ocorrem no documento dj. E fk,j, é a frequência do termo k no documento dj.

A frequência inversa do documento (IDF) foi proposta inicialmente por Jones (1972). A ideia por traz deste algoritmo é de que um termo de consulta que ocorre em muitos documentos não é um bom representante, e assim, deve ser dado menor peso do que os que ocorrem em apenas alguns documentos.

$$
i d f_{i}=\log \frac{N}{n_{i}}
$$

Onde $\mathrm{N}$ é igual ao número de documentos da coleção e ni é igual a quantidade de documentos em que o termo ocorre pelo menos uma vez.

A essência do funcionamento do método TF*IDF consiste em determinar o quão relevante uma palavra é em relação a um conjunto de documentos. As palavras que são comuns em um pequeno grupo de documentos, ou em apenas um documento, 
tendem a ter pesos TF*IDF mais elevadas do que as palavras comuns a todos os documentos.

Entretanto, o objetivo do uso da ponderação de termos neste trabalho, é de identificar os principais tópicos debatidos no fórum. Além do mais, com a extração das stopwords é possível eliminar grande parte dos termos que não apresentam significância ao contexto da discussão. Sendo assim para que se possa identificar as palavras-chave, faz-se necessário utilizar um algoritmo que atribua pesos mais significativos aos termos mais frequentes na coleção de documentos, ou seja, nos tópicos do fórum.

Pelo fato de dar menor peso aos termos que são frequentes dentro de um documento, mas não tão frequente na coleção, o TF*IDF não se torna adequado para a resolução do problema em questão.

Proposto em 2001 por Khoo Khyou Bun e Mitsuru Ishizuka, o TF*PDF (Term Frequency * Proportional Document Frequency) é uma abordagem que busca atribuir pesos mais significativos aos termos mais frequentes na coleção de documentos.

Em sua abordagem inicial, o algoritmo $\mathrm{TF}^{*} \mathrm{PDF}$ é usado para reconhecer os termos que explicam os principais temas de cada arquivo de notícias (Hot Topics) semanais. Sua proposta se baseia no conceito de que sempre que houver um hot topic "no ar", o tema será discutido com frequência em muitos documentos e fontes de notícias. Diferente da atribuição convencional de peso trabalhado no método TF*IDF, no algoritmo $\mathrm{TF}^{*} \mathrm{PDF}$, o peso de um termo é linearmente proporcional à frequência, e exponencialmente proporcional à relação do documento que contém o termo. Sendo assim o algoritmo PDF é representado pela fórmula a seguir:

$$
\text { PDF }=\exp (\mathbf{n i} / \mathbf{N})
$$

Desde sua proposta inicial em 2001 por Bun e Ishizuka o TF*PDF tem se demonstrado uma excelente ferramenta na mineração de texto para a detecção de tópicos em um documento ou em um conjunto deles. Diversos trabalhos nos últimos anos tem demonstrado a eficiência deste algoritmo como: (JAHNAVI; RADHIKA, 2012); ZHE et al., 2012); (REN et al., 2011); (KAUR ; GUPTA 2012); MA (2011), entre outros.

Sendo assim, este trabalho faz uso deste método como ferramenta de mineração de texto a fim de extrair os principais temas debatidos nos fóruns de aprendizagem.

Para se calcular a frequência do termo (TF) foi adotado uma abordagem diferente. Como não é feito cálculo de similaridade entre os documentos a coleção de documentos (os diferentes tópicos do fórum de discussão) são agrupados como um único documento e os pesos TF’s são calculados para todos os termos em relação a um único documento. Uma vez feito isto, o cálculo do peso PDF é realizado levando em consideração a frequência do termo em relação ao conjunto de documentos.

Uma vez que através do algoritmo TF*PDF os termos dos documentos são ponderados é realizada a ordenação deles para identificar quais os principais termos referenciados nesta discussão.

4. Uma vez feita a identificação dos principais tópicos discutidos no fórum, o sistema seleciona os cinco primeiros termos e os submeter a um motor de busca. No exemplo aqui proposto, foi utilizado a API do motor de busca Bing que oferece 5000 consultas gratuitas por mês, e permite que os links sejam retornados tanto em XML quanto em JSON. 
5. Após feita a consulta, os links (10 primeiros) são apresentados ao usuário como sugestão de leitura.

6. Um mecanismo de avaliação é proposto com o propósito de verificar a relevância do link ao fórum o qual foi proposto. O usuário poderá avaliar positivamente (gostei) ou negativamente (não gostei) de acordo com o enquadramento do documento em meio a discussão proposta no fórum.

7. Uma vez que o usuário gosta de um link recomendado, é feito um processo de indexação do campo descrição (que apresenta um resumo do documento) e guarda os termos de maior peso no perfil do usuário para que em um momento posterior possa fazer sugestões de outros materiais baseado neste seu interesse.

\section{A PESQUISA}

Com o objetivo de verificar a eficiência do algoritmo implementado neste trabalho, foi realizado uma pesquisa em onze diferentes disciplinas de três cursos técnicos oferecidos pela Universidade Estadual do Maranhão (UEMA) por meio do Núcleo de Tecnologias para Educação, UemaNet.

As disciplinas escolhidas para a pesquisa foram: Análise de Sistemas; Projeto de Redes; Interação Homem Computador; Gerência de Projetos; Programação Orientada a Objetos; Linguagem de Programação II; Sistemas Operacionais; Fundamentos de Informática; Aplicações Web I; Aplicações Web II e Projeto e Desenvolvimento de Sistemas.

O Núcleo de Tecnologias para Educação (UemaNet) presta suporte tecnológico à educação presencial e é responsável pela concepção, intermediação, gestão, avaliação e difusão de projetos em educação a distância da UEMA. A estrutura de gestão do UemaNet está organizada de forma descentralizada sendo que até o momento atende um total de 36 polos organizados para suporte às atividades acadêmicas e encontros presenciais. A Uema, por meio do UemaNet, atua junto à Secretaria de Educação Profissional e Tecnológica (SETEC), vinculando-se ao Programa Escola Técnica Aberta do Brasil (Rede e-Tec Brasil) com a oferta de cursos técnicos de nível médio a distância. Todas as disciplinas escolhidas para a realização da pesquisa aqui apresentada fazem parte da Rede e-Tec, que são os cursos: Técnico em Informática; Técnico em Redes de Computadores; e Técnico em Gestão em TI.

O objetivo desta pesquisa foi verificar a eficiência na extração automática de palavras- chave dos tópicos de discussão do AVA e averiguar se o uso destes termos proporcionou a recuperação de links relevantes ao tema em discussão.

Para esta averiguação, foram extraídos diversos tópicos (em média 15 tópicos) de cada disciplina, salvos em arquivos de texto (.txt) e submetidos a execução do algoritmo. O sistema por sua vez, fazia a extração de palavras chaves, as ordenava e submetias a seis palavras de maior peso para a API do motor de busca Bing, que retornava os links (os 10 primeiros) os quais seriam sugeridos.

Feito isto, foi gerado um formulário contendo a descrição da proposta do fórum, as seis palavras chaves de maior relevância segundo o algoritmo proposto, os links recomendados, e os tópicos utilizados para a execução dos testes. Para avaliar a eficiência, ninguém melhor do que os próprios professores que administravam as disciplinas. Desta forma, lhes foi encaminhado o formulário, contendo os itens descritos acima e mais duas perguntas:

1. As palavras- chave extraídas possuem representatividade em relação ao texto dos fóruns?

2. Os links sugeridos estão de acordo com o tema da discussão? 
Dos onze questionários elaborados e submetidos à avaliação dos professores, dez responderam que concordam completamente em ambas as questões e um respondeu que concorda parcialmente. Sendo assim, apesar da pequena amostra utilizada na pesquisa, pôde se verificar a eficiência da captura automática das palavras chaves e a consequente a apresentação de links relevantes à temática debatida no fórum.

Apesar do algoritmo ter se mostrado bastante eficiente na extração dos hot topics dos fóruns, através da implementação da pesquisa pôde se verificar algumas vulnerabilidades. Na disciplina em que o professor avaliou com "concordo parcialmente", foi identificado que o tema principal da discussão era "Sistemas Operacionais", entretanto, na maioria dos tópicos os alunos referenciavam apenas pela sigla "SO", desta forma, o processo remoção de stopwords acabava desconsiderando este termo. Outro problema verificado foi referente a flexão das palavras. Em algumas situações o algoritmo capturou a palavras no singular e também no plural.

\section{CONCLUSÃO}

Como já apresentado neste trabalho, a interação com o conhecimento e com as pessoas é fundamental para o processo de aprendizagem, sendo assim, os fóruns de discussão de um AVA buscam oferecer um meio pelo qual os seus participantes interajam de forma a transformar um determinado grupo de informações em conhecimento. As múltiplas interações e trocas comunicativas entre seus participantes possibilita que estes conhecimentos sejam reconstruídos e reelaborados. Entretanto, esta participação exige preparo através de leituras adequadas, permitindo que haja a troca de opiniões pessoais fundamentadas sem dar espaço ao "achismo".

O sistema proposto neste trabalho busca oferecer um meio automático de recomendar links para fomentar as discussões nos fóruns. O algoritmo TF*PDF proporciona um meio de extrair os tópicos mais importantes, levando em consideração que os termos mais relevantes aparecem com bastante frequência, sendo assim, este método difere do modelo clássico de ponderação de termo e pesos proposto por Salton (1988) ao dar pesos mais significantes aos termos mais frequentes.

Os testes preliminares do algoritmo aplicado a fóruns de discussão apontaram algumas lacunas que devem ser tratadas antes de colocar a ferramenta em um ambiente de produção. Entende-se que estes testes devem ser estendidos para ampliar a observação do comportamento do algoritmo e talvez encontrar outros problemas a serem tratados.

Futuramente pretende-se incorporar o algoritmo implementado em um plugin para o Moodle possibilitando testes mais ostensivos e a sua utilização em produção. Uma outra ideia, é conectar o mecanismo de busca (metabusca) a um repositório de objetos de aprendizagem, dando mais garantias sobre a qualidade dos itens recuperados.

\section{REFERÊNCIAS}

ARANHA, C. PASSOS, E. A Tecnologia de Mineração de Textos. RESI-Revista Eletrônica de Sistemas de Informação, n.2, 2006.

BUN, Khoo Khyou. ISHIZUKA, Mitsuru. Information Area Tracking and Changes Summarizing in WWW In: Proc. of WebNet 2001, International Conf. on WWW and Internet. Orlando, Florida, 2001.p.680-685.

HAN, J. KAMBER, M. Data Mining: Concepts and Techniques, 2nd ed. Morgan Kaufmann, 2006. 
JONES, Karen S. A statistical interpretation of term specificity and its application in retrieval. Journal of Documentation. v.28 1972. p. 11-21

JAHNAVI, Y. RADHIKA, Y. A Cogitate Study on Text Mining. International Journal of Engineering and Advanced Technology (IJEAT) ISSN: 2249 - 8958, v.1, Issue-6, aug. 2012.

KAUR, K. GUPTA, V. A Survey of Topic Tracking Techniques. International Journal of Advanced Research in Computer Science and Software Engineering. v.2, Issue 5, may. 2012

KENSKI, V. M. Processos de interação e comunicação mediados pelas tecnologias. In: ROSA, D., SOUZA, V. (Orgs.). Didática e práticas de ensino: interfaces com diferentes saberes e lugares formativos. Rio de Janeiro: DP\&A, 2002.

KOnCHADY, Manu. Text Mining Application Programming. Thomson. 2006.

KUECHLER, W. L. Business applications of unstructured text. Communications of ACM, v.50, n.10, p. 86-93, 2007.

LOPES, M. C. S. Mineração de Dados Textuais Utilizando Técnicas de Clustering para o Idioma Português. PhD thesis, Universidade Federal do Rio de Janeiro, 2004.

MA, HUI-FANG. Hot Topic Extraction Using Time Window. International Conference on Machine Learning and Cybernetics, Guilin, 10-13 ,July 2011.

PIVA, D. PUPO, R. GAMEZ, L. OLIVEIRA, S. EaD na Prática. Planejamento, métodos e ambientes de educação online. Rio de Janeiro: Elsevier, 2011.

OLIVEIRA, Gerson P. O Fórum em um Ambiente Virtual de Aprendizado Colaborativo. Revista Digital de Tecnologia Educacional e Educação a Distância.v. 2. ISSN 1808-1061, 2005.

REZENDE, S. O. MARCACINI, R. M. MOURA, M. F. O uso da Mineração de Textos para Extração e Organização Não Supervisionada de Conhecimento. Revista de Sistemas de Informação da FSMA n. 7 (2011) pp. 7-21

REN, Y. DU, Y. HUANG, X. XU, Y. Topic Detection of News Stories with Formal Concept Analysis. Journal of Information \& Computational Science 8: 9 (2011) 1675-1682.

RICCI, F. ROKACH, L. SHAPIRA, B. KANTOR, P. B. Recommender Systems Handbook .New York: Springer, 2011.

SCARINCI, R. G. SES: Sistema de Extração Semântica de informações. Porto Alegre, 1997. 165p. Dissertação de mestrado - Instituto de Informática, UFRGS.

SILVA, Marco. Educação online. São Paulo: Loyola, 2006.

SALTON, G. BUCKLEY, C. 1988. Term-weighting approaches in automatic text retrieval. Information Processing \& Management ,v.24, n.5, p. 513-523, 1988.

WIVES, L. K. Tecnologias de descoberta de conhecimento em textos aplicadas à inteligência competitiva. Exame de Qualificação EQ-069, PPGC-UFRGS, 2002.

ZHE, G. DONG, Li. QI, Li. JIANYI, Z. YANG, Xin. XINXIN, Niu. An Online Hot Topics Detection Approach Using the Improved Ant Colony Text Clustering Algorithm. Journal of Convergence Information Technology(JCIT),v. 7, n.2, February 2012. 